\title{
Artificial Intelligence for Social Good in Responsible Global Citizenship Education: An Inclusive Democratized Low-Code Approach
}

\author{
Meng-Leong How \\ Nanyang Technological University Singapore
}

\begin{abstract}
Although the world has become more connected than ever, inequality and poverty continue to hamper sustainable development. To surmount these challenges, UNESCO promotes Global Citizenship Education (GCED), which seeks to instill values, attitudes and behaviors in people to consider the importance of responsible global citizenship: creativity, innovation, and dedication to peace, human rights, and sustainable development. It allows students of all ages to understand that these questions are global, not local, so that they can actively promote peaceful, tolerant, inclusive, safe, and sustainable societies. This paper shows how a userfriendly low-code human-centric probabilistic approach can be used to democratize AI usage so that people who are not computer scientists may also use AI for social good through this inclusiveness.
\end{abstract}

\section{KEYWORDS}

inclusive; democratized; responsible education; global citizenship education; predictive simulations; Bayesian networks; no-code; low-code; artificial intelligence; optimizations; human-centric reasoning; human-in-the-loop; policy assessment

\section{INTRODUCTION}

Even though the world has become more connected, inequality and poverty are still detrimental to peace and sustainability. To overcome these challenges, UNESCO has been championing Global Citizenship Education (GCED), which seeks to instill ideals, attitudes and behaviors in people that promote responsible global citizenship: creativity, innovation, and dedication to peace, human rights, and sustainable development (Pigozzi, 2006). It enables learners of all ages to understand that these issues are global, not local. It encourages them to actively promote more peaceful, tolerant, inclusive, secure, and sustainable societies.

It is imperative to educate learners of all ages so that they can become empowered as responsible global citizens. Artificial intelligence (AI) is increasingly being used to contribute social good (Floridi et al., 2020). However, it remains challenging for educators or social science researchers who are not trained in computer science to write code and implement AI algorithms that can analyze datasets. The current paper demonstrates how the use of AI can be democratized with a user-friendly probabilistic approach to augment human thought. Analysts who are not computer scientists may also be empowered by this inclusiveness to use AI for predictive modeling of issues for social good. In education, this human-centric probabilistic approach may also be implemented by teachers as a cognitive scaffolding to encourage students 


\section{2nd World Conference on Teaching and Education}

to ask more questions which can contribute to the thought processes involved in becoming responsible global citizens.

\section{RESEARCH PROBLEM AND RESEARCH QUESTIONS}

For analysis of factors that contribute to global citizenship education, artificial intelligence (AI)-based approaches are helpful. There are also many initiatives to promote the use of AI for social good (Taddeo \& Floridi, 2018). However, the problem was, it was not easy for people with no computer programming skills to use AI to analyze data. Hence, the research questions that guide this paper are:

RQ1: How can people who are not trained in computer science also use AI to analyze data for social good?

RQ2: Is there an inclusive democratized low-code approach of using AI that does not require extensive computer software programming?

\section{METHODS}

An inclusive democratized low-code AI-enhanced approach can be used to create actionable predictive insights to contribute to social good. Three exemplars will be used to illustrate how an inclusive low-code human-centric probabilistic reasoning approach can be employed in the predictive modeling of social issues that are concerned with GCED. They are (1) global sustainable development, (2) ameliorating malnutrition, and (3) advancing financial inclusion for people who are unserved by traditional banking institutions.

Low-code tools enable rapid drag-and-drop development of predictive simulations, while allowing complete customizations of variables and artificial intelligence algorithms (Chang \& Ko, 2017). In effect, this user-friendly approach enables humans to focus on the allimportant big picture, logical reasoning, and productive collaborative discussions with stakeholders across multiple domain verticals via "what-if" scenarios for optimizations and risk assessments, while the AI-enabled software augments human ingenuity by doing the heavy lifting of making everything work smoothly in the simulations.

\section{DISCUSSION}

\subsection{Inclusive democratized low-code approach of using AI for social good: global sustainable development}

Sustainable development is essential to humankind (Griggs et al., 2013). The use of primary socio-environmental data for analysis is essential for informing policy makers on sustainable development decision making (see Figure 1). Using a low-code inclusive approach, non-computer scientists can also use AI to analyze Environmental Performance Index (EPI) data related to sustainability [authors, year]. This human-centered approach to probabilistic thinking may also be used as a cognitive scaffold to encourage analysts to ask additional questions and to provide decision-making assistance to inform sustainable development 


\section{2nd World Conference on Teaching and Education}

policymaking [authors, year]. The data from 180 countries in the EPI 2018 includes environmental health and ecosystem vitality performance indicators (Gupta \& Vegelin, 2016). Analysis using an inclusive and democratized no-code AI-enabled approach of the 2018 EPI data revealed hidden tensions between the two fundamental dimensions of sustainability: (1) the environmental health that improves economic growth and increase affluence, and (2) the vitality of ecosystems which worsens due to industrialization and urbanization.

Figure 1 to be inserted here.

\subsection{Inclusive democratized low-code approach of using AI for social good: ameliorating malnutrition}

Malnutrition, according to the World Health Organization (WHO) and the World Bank, is one of the world's most significant but least-addressed sustainability issues (Briend et al., 2006). Malnutrition refers to nutritional malfunction or imbalance, which could be influenced not only by under-nutrition, but also over-nutrition (Shrimpton \& Rokx, 2012). The double burden of malnutrition confers a serious and negative economic impact on individuals and populations. As the burden of malnutrition continues to rise, so too does its economic toll (Delisle, 2008). The double burden of malnutrition now facing many countries worldwide is characterized by the coexistence of undernutrition along with overweight, obesity or dietrelated noncommunicable diseases. While the double burden of malnutrition may pose a significant public health challenge, it also offers a unique opportunity for alignment and coordination for integrated action on malnutrition in all its forms. The identification of the double burden of malnutrition should be regarded as a catalyst for the achievement of key global goals for addressing policy and program interventions [authors, year].

AI can be democratized to allow non-computer science analysts to use human-centered explainable-AI to simulate the potential dynamics between malnutrition, health, and population indicators in a World Bank dataset collected from 180 countries. A rudimentary, beginnerfriendly Bayesian predictive modeling approach based on AI can be used to demonstrate how human-centered probabilistic reasoning can be used to analyze the dynamics of global malnutrition and optimize conditions to achieve the best-case scenario (see Figure 2). Conditions were also simulated for the worst-case scenario and could be used to inform stakeholders to prevent them from happening. Thus, it could improve the nutritional and health status of vulnerable populations. Malnutrition analysts, scientists, and policy makers - and not just computer scientists - can use AI also to develop predictive models for malnutrition, health, and population information. With this approach, different variables can be kept consistent while other variables could be modified to visualize "what if" scenarios to prevent adverse outcomes and to anticipate "at-risk" conditions. Policy makers can therefore potentially be better informed and take preventive actions to avoid the worst-case scenario.

Figure 2 to be inserted here. 


\section{2nd World Conference on Teaching and Education}

Vienna, AUSTRIA

\subsection{Inclusive democratized low-code approach of using AI for social good: financial inclusion}

Financial inclusion is, according to the World Bank, immensely important, as it contributes to poverty reduction and prosperity growth (The World Bank, 2020). In emerging markets like Asia, Africa and South America, financial inclusion is particularly relevant. It fosters affordable access to formal financial services that can boost economic growth and wellbeing. Many people most in need of financial inclusion, such as low-income families and micro-enterprises, have little to no access to financial services. Even if financial services are readily accessible, there is a need to increase awareness among the target groups to get buy-in.

The advancement of financial inclusion involves making basic financial goods and services available and affordable to individuals or small businesses, such as transfers, remittance, deposits, loans, and insurance. The increasing need for advancing inclusive financial solutions means AI can play a crucial role in allowing providers to better understand potential consumer needs (Fan \& Zhang, 2017). The problem that AI must resolve should be focused on the human customer rather than the product or on increasing revenue for the financial service provider (FSP). AI is uniquely able to help

FSPs understand the preferences of future customers because it can rapidly decipher data patterns which humans might not be able to. Access to financial inclusion can help households to prepare for unforeseen emergencies. Unfettered access to financial services, such as credit and insurance, can be used to grow small businesses. This may also lead to more spending in education and health care, thus enhancing the overall quality of life of those previously underserved by FSPs.

Access to inclusive financing is crucial, especially for the lower-income segment. Financial inclusion allows households with low-income to be more economically resilient (Yin et al., 2019). By making better use of AI to analyze the data, FSPs can versatilely adjust to the potential customers' different needs. The forecasts generated by AI could inspire FSPs to make recommendations by suggesting new directions and ideas for the potential customers (see Figure 3). FSPs can understand their clients and patterns better, and even identify new market opportunities using tools that help AI and people work together. FSPs understand how certain concepts resonate with certain segments of customers. This can lead to questions and hypotheses for further human creativity-motivated AI analysis. A major factor toward this future would be the willingness of FSPs to develop preconceived ideas about how successful it should look and how successful it could look. FSPs can use AI for the analysis of data to produce new findings, for example new data patterns, untapped channel possibilities or new target audiences. As AI detects incremental efficiencies, human analysts can raise questions which lead to changes in innovative strategies that better address the needs of potential clients. This can increase trust between potential customers and the FSP, which provides these innovative solutions. This could also drive the FSP towards a general transformation of the business. It may be empowering to move from a non-AI-based deterministic machine to a probabilistic AI-based machine that constantly explores many options. The upside is rapid discovery. FSP cultures that are open to change and invest in people and technology will enable their teams to be future-proof and will drive their enterprises towards successful transformation. 


\section{2nd World Conference on Teaching and Education}

\section{9-21 February, 2021}

Vienna, AUSTRIA

The use of AI can improve the flow of information between the FSPs and potential customers. Consequently, FSPs can extend credit or offer other financial services to microenterprises or individuals not supported by traditional banks due to lack of collateral. We are capable of developing and using AI to improve human conditions for social good. Although AI may be powerful, it is imperative for humans to take leadership in the use of AI, instead of being led by AI.

Figure 3 to be inserted here.

\section{CONCLUSION}

Proponents of AI for social good might have preferred to use predictive analysis and simulations of alternative variables combinations to simulate in-silico what could not be easily accomplished in the real-world. However, it was not always easy to write code to develop AI algorithms or to develop software. Using the AI-based BN approach provided in the current paper, a multitude of scenarios could be simulated to calculate the conditions for the best and worst outcomes of issues such as sustainable development, amelioration of malnutrition, and the advancement of financial inclusion to reduce poverty.

By deepening the use of conceptual abstraction, problem-solving heuristics and data analysis of issues that could contribute to social good, it enables learners of all ages to understand that these issues are global, not local, and it can encourage them to actively promote more peaceful, tolerant, inclusive practices. Using user-friendly software applications, for example, Bayesialab, or other BN software, such as GeNie by BayesFusion, or Netica by Norsys, or Bayes Server, the examples in the current paper could be adapted by AI for social good analysts using their own data at the regional, country, or global level. The user-friendly inclusive low-code human-centric reasoning approach offered in this paper can democratize the use of AI by making it accessible to multidisciplinary analysts who may not be computer scientists. Moreover, AI-augmented thinking can transform how AI could be used cognitively and lead to more data explorations via AI and more human-centric insights for informing policymaking.

\section{REFERENCES}

Briend, A., Prudhon, C., Prinzo, Z. W., Daelmans, B. M. E. G., \& Mason, J. B. (2006). Putting the Management of Severe Malnutrition Back on the International Health Agenda. Food and Nutrition Bulletin, 27(3_suppl3), S3-S6. https://doi.org/10.1177/15648265060273S301

Chang, Y.-H., \& Ko, C.-B. (2017). A Study on the Design of Low-Code and No Code Platform for Mobile Application Development. International Journal of Advanced Smart Convergence, 6(4), 50-55. https://doi.org/10.7236/IJASC.2017.6.4.7

Delisle, H. F. (2008). Poverty: The Double Burden of Malnutrition in Mothers and the Intergenerational Impact. Annals of the New York Academy of Sciences, 1136(1), 172-184. https://doi.org/10.1196/annals.1425.026 


\section{2nd World Conference on Teaching and Education}

\section{9-21 February, 2021}

Vienna, AUSTRIA

Fan, Z., \& Zhang, R. (2017). Financial Inclusion, Entry Barriers, and Entrepreneurship: Evidence from China. Sustainability,

$$
\text { 9(2), } 203 .
$$

https://doi.org/10.3390/su9020203

Floridi, L., Cowls, J., King, T. C., \& Taddeo, M. (2020). How to Design AI for Social Good: Seven Essential Factors. Science and Engineering Ethics, 26(3), 1771-1796. https://doi.org/10.1007/s11948-020-002135

Griggs, D., Stafford-Smith, M., Gaffney, O., Rockström, J., Öhman, M. C., Shyamsundar, P., Steffen, W., Glaser, G., Kanie, N., \& Noble, I. (2013). Sustainable development goals for people and planet. Nature, 495(7441), 305-307. https://doi.org/10.1038/495305a

Gupta, J., \& Vegelin, C. (2016). Sustainable development goals and inclusive development. International Environmental Agreements: Politics, Law and Economics, 16(3), 433-448. https://doi.org/10.1007/s10784-016-9323-z

Pigozzi, M. J. (2006). A UNESCO view of global citizenship education. Educational Review, 58(1), 1-4. https://doi.org/10.1080/00131910500352473

Shrimpton, R., \& Rokx, C. (2012). The Double Burden of Malnutrition: A Review of Global Evidence. World Bank.

https://doi.org/10.1596/27417

Taddeo, M., \& Floridi, L. (2018). How AI can be a force for good. Science, 361(6404), 751-752. https://doi.org/10.1126/science.aat5991

The World Bank. (2020). Financial Inclusion. https://www.worldbank.org/en/topic/financialinclusion/overview

Yin, X., Xu, X., Chen, Q., \& Peng, J. (2019). The Sustainable Development of Financial Inclusion: How Can Monetary Policy and Economic Fundamental Interact with It Effectively? Sustainability, 11(9), 2524. https://doi.org/10.3390/su11092524 


\section{2nd World Conference on Teaching and Education}

19-21 February, 2021

Vienna, AUSTRIA

WORLDCTE

World Conference on

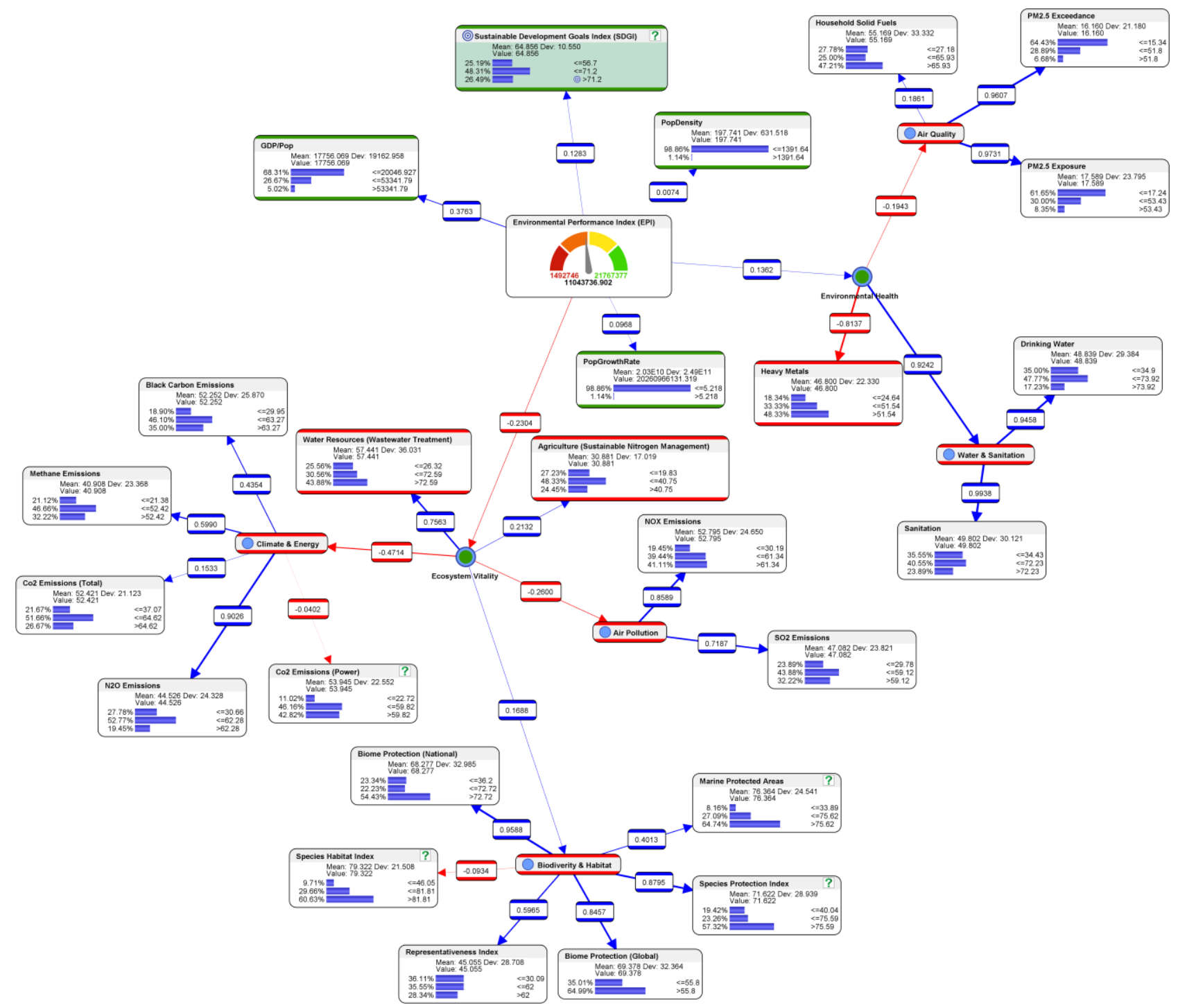

Figure 1. AI for social good: Bayesian predictive modeling for analysis of global sustainable development 


\section{2nd World Conference on Teaching and Education}

19-21 February, 2021

Vienna, AUSTRIA

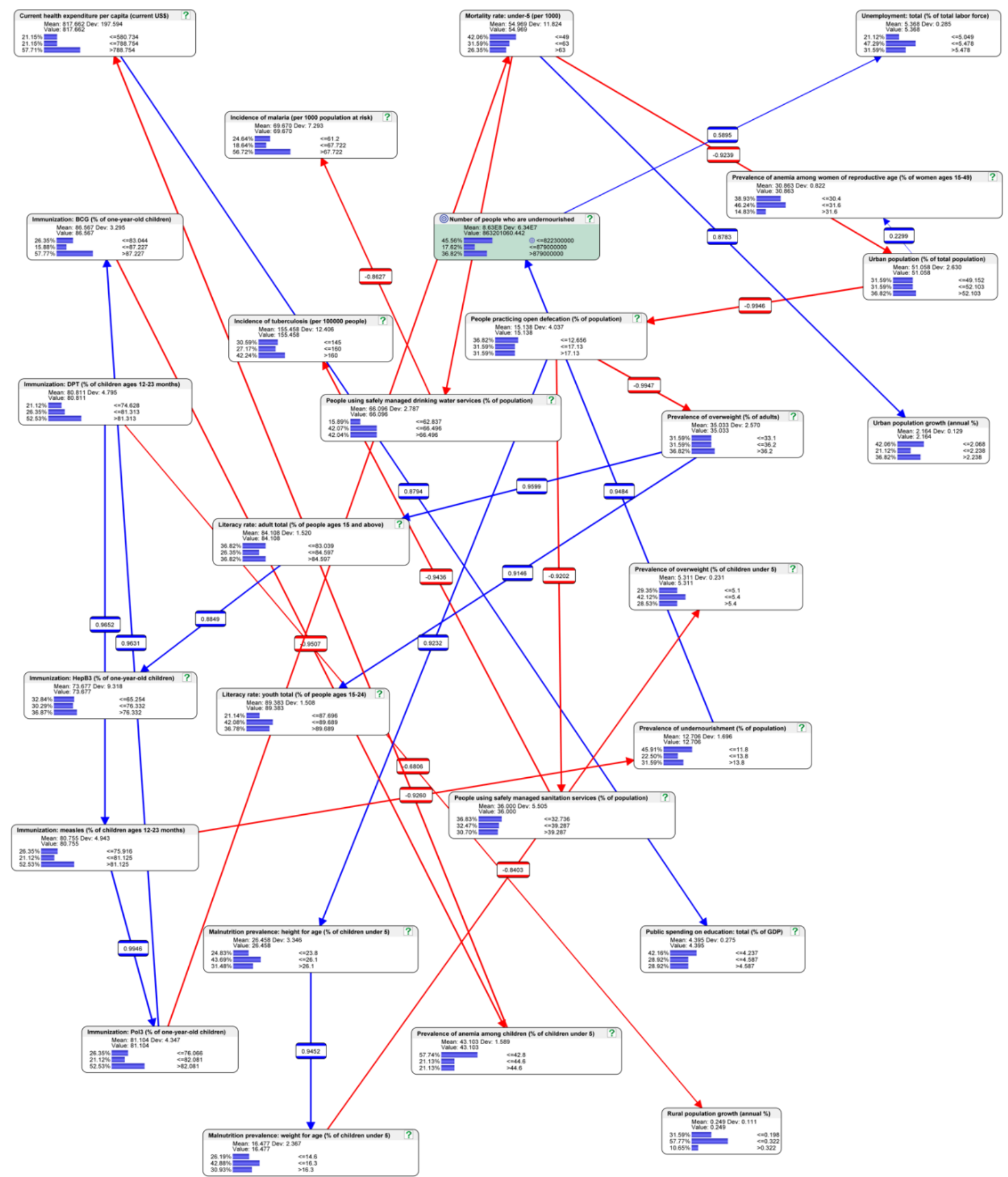

Figure 2. AI for social good: Bayesian predictive modeling for ameliorating global malnutrition 


\section{2nd World Conference on Teaching and Education}

19-21 February, 2021

Vienna, AUSTRIA

WORLDCTE

World Conference on

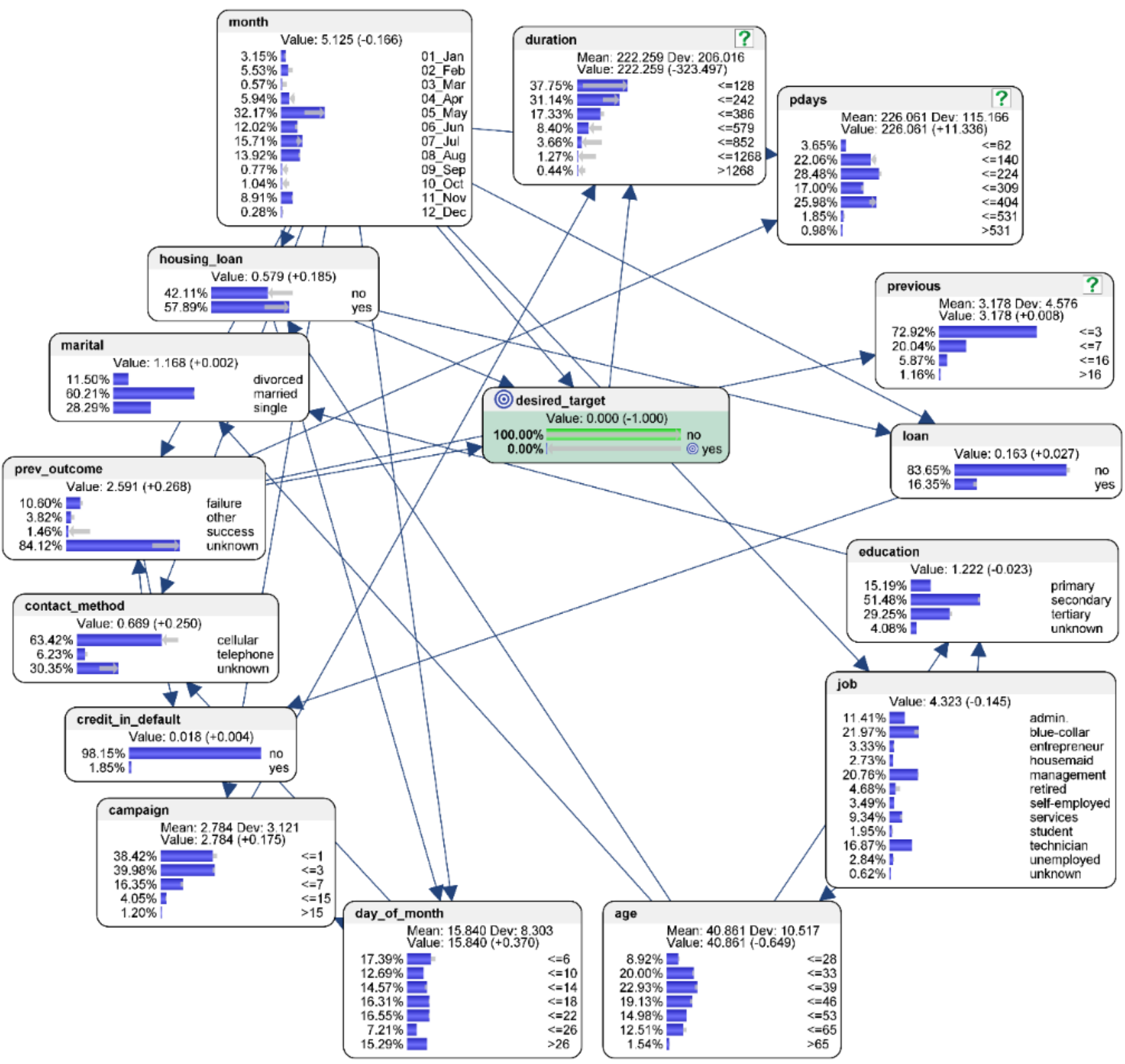

Figure 3. AI for social good: Bayesian predictive modeling for advancing financial inclusion and poverty reduction 